\title{
Effects of a Biomechanical-based Tai Chi Program on Gait and Posture in People With Parkinson's Disease: Study Protocol for a Randomized Controlled Trial
}

Nok-Yeung Law ( $\square$ nlaw098@uottawa.ca )

University of Ottawa https://orcid.org/0000-0001-7390-7509

Jing Xian Li

University of Ottawa

Qingguang Zhu

Shanghai University of Traditional Chinese Medicine

Julie Nantel

University of Ottawa

Study protocol

Keywords: Parkinson's disease, Gait, Postural stability, Falls, Tai chi

Posted Date: June 17th, 2021

DOI: https://doi.org/10.21203/rs.3.rs-412472/v1

License: (9) This work is licensed under a Creative Commons Attribution 4.0 International License.

Read Full License 


\section{Abstract}

\section{Background}

Parkinson's disease (PD) is associated with changes in gait and posture that can lead to a higher frequency of falls and injuries in this population. Research has shown a positive effect of tai chi (TC) training on the movement capacity for those with PD, however the understanding of the impact of TC training on gait and postural stability in PD is lacking. This study aims to examine the impact of a biomechanical-based TC training on dynamic postural stability and how it relates to walking performance.

\section{Methods/Design}

We will conduct a prospective, single-blind, randomized control trial of 40 individuals with early-stage PD (Hoehn \& Yahr stages 1 to 3). Those with PD will be randomly assigned to either a TC group or a control group. The TC group will participate in a biomechanical-based TC training program that is formed based on the movement analysis of TC and will be practiced two to three times a week for 12 weeks. The control group will be asked to engage or maintain their regular physical activity (PA) for a period of 12 weeks. The primary and secondary outcomes will be assessed at baseline, 6-week, and 12-week after commencing the study protocol. The primary outcome measures will include gait speed, cadence, step length during level surface walking (simple task) and fixed-obstacle crossing (challenging task); the dynamic postural stability will be indicated by the center of mass and center of pressure (COM-COP) separation distance and clearance distance measured during fixed-obstacle crossing. The secondary measures will be the Unified Parkinson's disease Rating scale (UPDRS-III), single leg-stance test with eyes open and closed, and three cognitive scores (Stroop Test, Trail Making Test- Part B, and the Wisconsin Card Sorting Test).

\section{Discussion}

This protocol could lead to the development of an original and innovative TC training program for improvement of gait and postural stability among individuals with PD, which could help to improve or preserve the participants' self-confidence, active participation in social activities, and therefore be beneficial to their overall quality of life.

Trial registration: ClinicalTrials.gov (registry), NCT04644367 (registration number), November 25, 2020 (date of registration).

\section{Introduction}

\section{Background and rationale $\{6 a\}$}

Parkinson's disease (PD) is a growing concern in Canada as it is estimated that over 100,000 Canadians are living with PD, this number is expected to rise to 163,700 by $2031[1,2]$. The socioeconomic costs 
associated with this neurological condition is estimated to be $\$ 558.1$ million, with $70.2 \%$ of this amount going toward long-term disability care [3]. Postural instability and gait difficulties are common problems reported among those with PD [4-10]. Those with PD have difficulties with daily tasks such as walking due to gait-related changes in step width and step length that can lead to reduced walking speeds $[11,12]$. This "cautious gait" approach is similar to older adults who are at a high risk of falls [13-16]. Research has demonstrated that falls and the loss of ability to live independently are key concerns facing those with PD $[17,18]$. Reported falls occurrence in PD is $68.3 \%$ over a 1 -year period [19].

Currently, exercise intervention programs have been used in conjunction with drug therapy (such as Levodopa, carbidopa) as standard of care to manage the symptoms related to PD [20-23]. TC is a bodymind exercise that has been recommended by Parkinson's Canada as one of the few exercise programs specific for those with PD [24,25]. TC training is suggested to help improve awareness of whole-body movement and joint proprioception [26-28], increase muscle strength and endurance of the lower limb [29-31] that in turn would strengthen the neuromuscular reaction of muscles in the lower extremities $[32,33]$. For those with $\mathrm{PD}, \mathrm{TC}$ training would help to challenge postural stability, particularly in the mediolateral (M-L) direction that is closely related to fall prevention [34-37], and strengthen the muscles at the hip involved in postural control. Regular TC practice and training would lead to improvements in gait and postural stability by encouraging weight shifting, control displacement of the COM over the base of support, and comfort with stepping in different directions.

\section{Objectives $\{7\}$}

Previous TC studies for PD did not indicate how their programs were formulated or how their TC forms were selected [38,39], which can affect the training effect as TC has many forms and has different styles. Moreover, these studies did not consider the impact of TC training on dynamic postural stability and how it relates to walking performance and challenging walking, such as obstacle crossing. This can be an especially difficult for those with PD as dynamic postural stability is challenged. This study aims to examine the impact of a biomechanics-based TC program training on gait and postural strategies, particularly on dynamic postural stability and how it relates to walking performance and obstacle crossing. Based on our previous studies $[27,29,31,40]$, it is hypothesized that a biomechanics based TC program training will help to improve gait and dynamic postural stability by increasing walking speed, stability in mediolateral (M-L) direction, and foot clearance distance in individuals with PD. This protocol could lead to the development of an original and innovative balance and gait training program tailored to individuals with PD.

\section{Trial design $\{8\}$}

A single-blind randomized control trial will be conducted to compare the effects of a TC program with a typical PA routine (figure 1). The study period will be 12 weeks (3-months) that will include a 12-week intervention period with primary and secondary outcomes measured at baseline, 6 and 12 weeks. 


\section{Methods}

\section{Participants, interventions and outcomes}

\section{Study setting $\{9\}$}

The primary site for conducting the study will be at the University of Ottawa's Human Movement Biomechanics Laboratory. TC interventions will be offered at the community centers in Ottawa, Canada. The PA routine can be performed inside or outside the home environment.

\section{Eligibility criteria $\{10\}$}

Participants will be included in the study if they meet the following criteria:

1. Have been diagnosed with PD and demonstrate a disease severity ranging from 1 to 3 on the Hoehn and Yahr (H\&Y) scale [41]

2. Have no fluctuations in motor symptoms as reported by the motor section of the Unified Parkinson's Disease Rating Scale (UPDRS-III) [42]

3. Have stable medication use

4. Can stand and walk independently

5. Availability: three times per week over a period of 12 weeks

Participants will be excluded from the study if any of the following conditions are reported:

1. If they are enrolled into any other behavioral or pharmacological studies

2. Have a Montreal Cognitive Assessment (MoCA) score of $<26 / 27$ (that indicate some mild cognitive impairment (MCI)) [43]

3. Have had a serious medical condition or uncontrolled hypertension (equal or greater than a systolic 180 or diastolic 110 reading)

4. Have any debilitating conditions that could prevent them from performing any PA for a minimum of 60 minutes

\section{Who will take informed consent? $\{26 \mathrm{a}\}$}

The primary investigator will obtain informed consent from the potential trial participants.

Additional consent provisions for collection and use of participant data and biological specimens $\{26 \mathrm{~b}\}$

$\mathrm{n} / \mathrm{a}$

Interventions

Explanation for the choice of comparators $\{6 \mathrm{~b}\}$ 
The two comparators are the TC intervention group and PA (control group). The two comparators were chosen to understand the impact those with PD who are trained in TC compared to those who will be asked to maintain their regular PA. An active PA (control) group was chosen to avoid an unfair comparison to participants who are sedentary or who are inactive.

\section{Intervention description \{11a\}}

\section{TC program development}

The biomechanical-based TC training program has been developed by working with an experienced TC master and by biomechanical analysis of TC movement together. Seven TC movements were chosen based on consideration of the following:

\section{Easy to learn}

2. Feasible to practice

3. Can be practiced either individually or in groups

4. Space requirement is minimal, for example, practicing inside the home, living room or a yard

Biomechanical characteristics of TC movements. The TC program includes seven movements, three relatively static and four dynamic forms, which are listed in Table 1. Figure 2 illustrates three dynamic TC movements from the seven TC movements. These TC movements are typical TC movements and are present in all classic TC styles such as Wu, Yang, and Chen styles [46]. The biomechanical analysis of the selected seven TC movements, in terms of their kinematics, kinetics, and muscle activities, demonstrates their unique features compared to walking. Performing these movements requires increased foot clearance, step length, and movement in the backward, sideway, 45 degree angle, and forward directions $[27,44]$. Regular performance of these movements would result in special training to postural control, particularly in the adductor and tensor fasciae latae muscles that are responsible for maintaining M-L postural stability and assisting in preventing falls $[27,29,45]$. Our previous studies have shown that TC training can help to challenge M-L stability through shifting of the body's COM, utilizing the full range of motion (ROM) of joints, and increasing step width $[27,29]$. Electromyography activity while performing the seven TC forms has revealed a higher activation of the hip adductor muscles compared to walking that suggests good training to the muscles related to prevent lateral falls [47].

Table 1. 7-form TC program for people with PD. 


\begin{tabular}{|ll|}
\hline TC form & Characteristics \\
\hline Form 1: Wave-Hand-like-Cloud & Sideway stepping: left and right \\
\hline Form 2: Brush Knee Twist Step & Forward Diagonal stepping: left and right \\
\hline Form 3: Repulse Monkey & Backward Diagonal stepping: left and right \\
\hline Form 4: Part the Wild Horse's Mane & Lateral Diagonal stepping: left and right \\
\hline Form 5: Starting Form & Standing (arms): arms from down to up \\
\hline Form 6: Hero Lift the Sky & Standing (arms): push up to down \\
\hline Form 7: Standing, push hand back & Standing (arms): neutral to back \\
\hline
\end{tabular}

\section{TC training group}

For the TC group, the first two weeks of the program will require the participants to work on some light breathing and simple upper-limb forms; the basic TC forms will be introduced with a focus on becoming comfortable with the flow and rhythm of the movements. Each session will be 60 minutes in length and supervised by a TC instructor in a community center. The training will be gradual and range from low-tomoderate intensity. Participants will take 5 to 10 minutes to warm-up, 40 minutes of core activities, and a 5-minute cool-down. Three sessions will be offered weekly for 12 weeks; the participants will be asked to attend at least 2 sessions per week. The third to sixth week of the program will focus on incorporating the upper- and lower-limb into the routine; the focus of these classes will be to practice the seven TC forms sequentially. The remaining six weeks of the program will focus on repetitions and performance mastery of the basic seven-form routine. The participants will be allowed to practice TC at home. Any TC that the participant performs in their home or on their own time will be recorded by themselves using a monitoring form that will be distributed to them and collected weekly. The TC instructor will be present at each of the session to monitor the participants' progress and to ensure that the movements are being performed correctly.

\section{Control group}

The control group will track their level of PA over 12 weeks. During this period, the participants will be required to engage in at least 60 minutes of regular PA on their own, three times per week. Regular PA may include such activities as walking, cleaning or performing chores inside the home, and/or climbing the stairs; no restriction will be made to limit others forms of PA; individuals in the control group will be permitted to engage in organized sports, instructor-led class such as boxing, dance, etc. to ensure the participant recruitment process is feasible. The reason or value of using an active control group is for comparisons between one group who will receive formal TC training and the other who will not, and also to reflect the everyday lifestyle and activities of a typical individual with PD from the community. Similar to the TC group, the participants in this group will be asked to complete an activity log form for monitoring that will be collected weekly to monitor their regular PA levels. 


\section{Criteria for discontinuing or modifying allocated interventions $\{11 \mathrm{~b}\}$}

At any point during the study, either the intervention period or laboratory testing sessions, the participants will be free to refuse to participate. If he or she chooses to participate, the participant may withdraw from the study at any time for any reason. The criteria for discontinuing includes any minor injuries that may occur during the laboratory testing, class session, or outside of class (in the participants' home) such as fractures that do not require medical attention, muscle soreness or pain that persist for more than 48 hours, dizziness or faintness, or hypertension or hypotension that occur while exercising.

\section{Strategies to improve adherence to interventions $\{11 \mathrm{c}\}$}

For the group receiving the TC intervention, three class sessions will be offered weekly for 12 weeks; the participants will be asked to attend at least 2 sessions per week. These classes will be monitored by the $\mathrm{PI}$ and TC master. This will ensure that the classes are offered at a flexible time for the participant that match their availability and schedules. For the control group who will be asked to maintain regular PA levels, the participants of this group will be asked to complete an activity log form for monitoring that will be collected weekly to monitor their regular PA levels. This will help to ensure that the participants in the PA group will be motivated and consistent in their PA engagement and performance.

\section{Relevant concomitant care permitted or prohibited during the trial $\{11 \mathrm{~d}\}$}

There are no concomitant care and interventions requirements that are either permitted or prohibited during the trial. The participants will be asked to maintain their medication dosage, and any mandatory medical care or aid that they are receiving during the duration of the intervention period. To encourage engage and enrollment into the study, the TC and PA groups will be allowed to engage in forms of exercise and PA, no restrictions will be made as this will not affect the outcome or results of the study.

\section{Provisions for post-trial care $\{30\}$}

$\mathrm{n} / \mathrm{a}$

\section{Outcomes $\{12\}$}

All outcome measures will be conducted by the main research assistant, blind to group allocation, at 1 week (baseline), 6 weeks (mid-intervention), and 12 weeks (post-intervention). Demographic information will include age and sex of the participants. Clinical information will be obtained from the participants' reported health status and medical records from their hospital or healthcare providers.

\section{Primary outcome measures}

Gait performance and dynamic postural stability

1. Gait speed. This is a measure of how quickly an individual can walk within a specified distance. Gait speed is a global measure of functional capacity, mobility, and movement performance. 
2. Cadence. This measure is defined as the total number of full gait cycles that is completed over a period of time. This gait cycle, in human movement analysis, is defined as the repeated instances of subsequent foot contacts of the same foot. Cadence can reflect the quality of the timing and frequency of the movement; a good stepping or walking cadence can reflect the individual's movement capacity and their body's ability to maintain rhythmicity during movement.

3. Step length. This measure is defined as the distance travelled by a person over one cycle from the initial heel strike to the next heel strike.

4. COM-COP separation distance [48], in the anterior-posterior (A-P) and M-L directions. The separation distance of the body's COM and COP will be determined through motion analysis of walking and obstacle crossing trials and will be used to reflect the body's dynamic postural stability.

5. Clearance Distance $[49,50]$. The clearance distance will be measured from position of the foot to the height of the obstacle during obstacle crossing $[51,52]$. It is one of the indicators of the dynamic postural stability.

\section{Secondary outcome measures}

Clinical and Cognitive (Executive Function) assessment

1. Unified Parkinson's Disease Rating Scale- motor examination (UPDRS-III) [53]. This is a standard clinical assessment tool used to evaluate the severity and progression of the motor-related symptoms of PD. This scale has 14 -items, each scored on a 5-point Likert scale ranging from 0 to 4 , with 0 representing "no impairment" and 4 representing "marked impairment".

2. Single-leg stance test with eyes open (SLO) and closed (SLC). The participant will be asked to stand for as long as possible up to 60 seconds, with either eyes open or closed. Trials will be terminated if the participant loses his or her balance, or if his or her feet are moved from the initial position. The sequence of the eyes open or closed trials will be randomized. A total of three trials will be collected, with three-minute rest in between. This is selected because this test can be used to reflect the muscle strength of the lower extremities and to test static postural stability $[54,55]$.

3. Timed Up and Go Test (TUG). The individual will be instructed to stand up from a chair, walk 3 meters as quick and safely as possible, cross a line marked on floor, turn around, walk back, and sit down [56]. The individual will be permitted to use the chair's arm or back support for assistance when standing up, if needed [57]. The time that he or she takes to complete this task will be recorded starting from the time of the instruction "go". This is a simple test to measure functional mobility used in the clinical setting.

4. Trail Making Test Part B (TMT-B). The participants will be given a set of numbers and letters that they will be asked to connect with lines the numbers to letters in ascending order. The time that it takes the participant to complete this task will be recorded. The TMT-B is used to measure processing speed and mental flexibility [58-60], and as a screening tool for neurological deficits such as $\mathrm{MCl}[61,62]$. 
5. Wisconsin Card Sorting Test (WCST). The participants will be given a deck of 64 -cards and will be asked to sort the cards by shape, color, or number. The number of errors made, and number of successful sorted categories (maximum of 6 ) will be recorded. Participants will receive feedback whether the card sorted is "correct" or "incorrect"; after completing 10 consecutive sorts, the participants will switch to the next sorting category and this repeated until all the cards in the deck have been sorted. This is a frequently used measure of mental switching and response preservation [63].

6. Stroop Test. The participants will be asked to name the color but not to read the word on a card. A color-word score will be given based on the total correct identification of the correct color within 45 seconds. The Stroop Test is a measure of how the brain process interfering signals. This test measures attention, inhibition control, and response inhibition $[63,64]$.

\section{Participant timeline $\{13\}$}

\section{Sample size $\{14\}$}

The estimated number of participants needed to achieve the study's objectives is 40 participants.

According to Cohen (1988), the approximate number of participants needed in each group to attain $80 \%$ power in a difference between two independent means testing hypotheses at a 0.05 significant level is 24 for a comparison between the measurements of different time points if the estimated effect size is larger than 0.83 . Considering $10 \%$ of dropout rate of the participants recorded from a previous 24 -week TC intervention study for those with PD [38], it is estimated that there will be a $5 \%$ dropout rate in phase 3 of the study. Therefore, 22 will be recruited for young and elderly group respectively for the obstacle crossing study and 30 for the TC and regular PA group for the PD intervention program.

\section{Recruitment $\{15\}$}

Participants with PD will be recruited in the city Ottawa and greater Ottawa area, Ontario. Participation in the TC intervention will be on a "first-come-first serve" basis. The recruitment of the participants will be obtained through three sources: (1) referrals from local medical clinics (i.e., physician or neurologist offices); (2) local Parkinson's support groups that are associated with Parkinson's Canada; (3) bulletin board, newsletter or postings in the hospital.

\section{Assignment of interventions: allocation}

\section{Sequence generation $\{16 \mathrm{a}\}$}

Potential participants will be screen on the telephone by a research assistant. Those who are willing and eligible to participate will be randomly assigned in a 1:1 ratio to TC group or PA group using a concealed block-randomization procedure, stratified by age and sex. 
The block-randomization design will use permuted block sizes of 4 and 6 with the participant assignments placed in opaque, sealed and serially numbered envelopes and individualized for each participant.

\section{Implementation $\{16 \mathrm{c}\}$}

The randomization schedule that will be generated by the data analyst, who will deliver this document in a sealed envelope to the research assistant who will be responsible for assigning the participants to the two groups.

\section{Assignment of interventions: Blinding}

\section{Who will be blinded $\{17 \mathrm{a}\}$}

Only the participants will need to be blinded because the aim of this study is not behavioral-based modifications, rather to assess the gait and cognitive performance changes using measures obtained from standard biomechanical and neuropsychological assessment tools and testing procedures. Changes in the participants' gait and cognitive performance will be evaluated from their individual baseline scores; this will ensure that the effects of the two intervention programs are examined, not differences in the individual performance or expectations of the participants.

\section{Procedure for unblinding if needed $\{17 b\}$}

$\mathrm{n} / \mathrm{a}$

\section{Data collection and management}

\section{Plans for assessment and collection of outcomes $\{18 \mathrm{a}\}$}

A ten-camera Vicon motion analysis system (two Vicon Vantage and eight Vicon Bonita optical cameras, Oxford Metrics, Oxford, UK) with a sampling rate of $200 \mathrm{~Hz}$ will be used to capture the participants' gait and obstacle crossing. Prior to data collection, 45 reflective markers (14 mm diameter) will be placed on the participants' body landmarks according to a modified Helen Hayes marker set. Ground reaction forces will be collected using four force plates recorded at $1000 \mathrm{~Hz}$ (two Kistler models: Kistler Instruments Corp, Winterthur, Switzerland; two Bertec models: Bertec Corporation, Columbus, $\mathrm{OH}, \mathrm{USA}$ ). The force plates will be embedded in the middle of an 8-m long walkway. The participants from the TC and PA (control) groups will be required to visit University of Ottawa's Human Movement Biomechanics Laboratory on three laboratory sessions: at baseline, at week 6 , and week 12 intervention. The data collection session will consist of preliminary screening questionnaire, gathering of anthropometric data, 3-D motion capture during obstacle crossing and walking, and neuropsychological assessment of executive functioning.

\section{3-D motion capture:}


1. Gait performance test: Participants will be asked to walk along the 8-m long walkway at their selfselected comfortable speed during which their movement will be recorded using three-dimensional (3-D) motion capture for analysis of their 3-D kinematics and kinetics characteristics of the gait. Walking performance will be used as a baseline condition for comparison with obstacle crossing because it is a simple task to perform and can provide some indications to the participants' overall health and functional status. A total of five successful walking trials will be collected.

2. Obstacle crossing test: Participants will perform obstacle crossing in the laboratory for analysis of their dynamic postural stability and gait performance. An obstacle composing of two upright upholders and a crossing plank $20 \mathrm{~cm}$ high will be placed in the middle of the walkway where the force platforms are embedded. This plank has been made easily collapsible to keep the participants safe so that they will not fall. A total of five successful obstacle crossing trials will be collected.

\section{Neuropsychological Assessment.}

The following three neuropsychological assessment tools to examine executive functioning include: Trail Making Test Part B (TMT-B), Wisconsin Card Sorting Test (WCST), and Stroop Test. These three neuropsychological assessments tools are the three most commonly used measure of executive functioning in aging research and have been recommended as popular screening tools for assessment of mild cognitive impairment $[65,66]$.

1. TMT-B: participants are given a set of numbers and letters and are asked to connect a line from a number to a letter in ascending order. The time that it takes the participant to complete this task will be recorded.

2. WCST: participants will be given a deck of 64-cards and will be asked to sort the cards according to shape, color, and number. The number errors made, and number of successful sorted categories (maximum of 6) will be recorded. Participants will receive feedback whether they have sorted "correct" or "incorrect"; after completing 10 consecutive sorts, the participants will switch to the next sorting category and this repeated until all the cards in the deck have been sorted.

3. Stroop Test: participants are asked to name the color but not to read the word on a card. A color-word score will be given based on the total read in 45 seconds.

For the 3-D motion capture, the data will be collected and analyzed using Vicon's Nexus software ( $v$. 2.8.1) and tabulated in MATLAB (v. 7.1. Release 2006a) to obtain the temporal and spatial parameters. The functional and neuropsychological assessment measures will be gathered and stored both as hard and digital copies for further data interpretation and analysis. Descriptive analysis of the movements will be first conducted. We will check that all trials are completed and that there are no trials missing. For the 3-D motion capture data of the participants' movement, the data will be digitized, filtered, and processed. All Nexus software calculations will be derived from the Plug-in-gait model (Vicon Oxford Metrics, Oxford, UK). For each trial that will be collected, the data will be normalized to one full gait cycle. One gait cycle is defined as the succession of repeated foot-strike to the next foot-strike of the same foot. The primary outcomes will be normalized by the participants' body height and weight. 


\section{Plans to promote participant retention and complete follow-up \{18b\}}

Participants in the TC group will be asked to partake in minimum of two 1-hour TC class per week for twelve weeks. This duration and frequency of practice has previously been used in past TC studies for this population to achieve the desired beneficial effects on postural stability and movement capacity [67-70]. The selected intensity, duration, and frequency of the training is appropriate to meet the demands of a low-to-moderate level of exercise classification [71].

To mitigate any feelings of physical discomfort or muscle fatigue, the participants will be instructed to warm-up prior to the training and will be asked to perform each TC form at their preferred comfortable speed. The participants will be allowed to rest or take breaks when they prefer. Although it is unlikely, minor injuries may occur in some circumstances such as muscle pull, overstretching, or overstepping if the participants are unfamiliar with the TC forms. Any minor injuries will be treated properly with the necessary medical attention starting with the RICE approach (Rest, Ice, Compress, and Elevation).

The safety of the participants in the community center and laboratory settings is a primary concern of the researchers. At all times during training session or laboratory testing, both the $\mathrm{TC}$ instructor, $\mathrm{Pl}$, and/or a research assistant(s) will be present to monitor the performance and abilities of each individual participant. Those who are deemed to be at risk of falling will be provided with a spotter who will ensure the safety of the participant. Monitoring by the PI, instructor and/or research assistant will help to prevent accidental trips and falls. During the TC session, it is important to monitor the participants' progress in the TC program. To encourage compliance in the program, the PI will confer with the participants' daily activity log of their TC practice to see if they are achieving the weekly goals and objectives for the class.

Should any adverse events occur during TC training session, efforts will made to quickly identify this concern. The participants will be free to refuse to participant and, if he or she chooses to participate, the participant may withdraw from the study at any time for any reason. A major adverse event is any event that causes serious or permanent adverse outcomes such as death or leading to hospitalization. A minorto-moderate adverse event is any minor injuries such as fractures that do not require medical attention, muscle soreness or pain that persist for more than 48 hours, dizziness or faintness, or hypertension or hypotension that occur while exercising. All major and minor adverse events that may occur during laboratory testing, a class session or outside of class (in the participants' home) will be carefully documented in the project logs. This incident report will be passed immediately to the Research Ethics Board (REB) of the University of Ottawa and to our supported funding boards such as Parkinson's Canada.

\section{Data management $\{19\}$}

The data (completed informed consent form, testing notes, electrical testing data and printed testing data) will be carefully filed and securely stored in the researcher's office at the University of Ottawa. The computer in the researcher's office will also be locked to limit the access. The data will be encrypted and stored in the password protected and encrypted computer of the researcher. The computer will be locked 
to limit the access. A number code system will be employed during the data collection, analysis, and reporting. In addition, only can the researchers access the number code system in relation to the data obtained from the participants (e.g., electronic data, the master sheet). The master sheet linking the participants' identification to their number code and other data documents will be kept in a locked filing cabinet of the Pl's office.

\section{Confidentiality $\{27\}$}

Information from the study will be held in strict confidentiality and will not be used except for research purpose. The information collected in the study will be coded in a way that will ensure anonymity. Any published results will be presented with complete anonymity. Any personal data (e.g., address, telephone number) and testing data will be kept in a separate file folder, accessible only by the researchers responsible for the project. The data will be conserved for a period of 5 years after the time of publication. After the time of publication, all the electronic data will be deleted from the computer entirely. The printed data file will be shredded.

\section{Plans for collection, laboratory evaluation and storage of biological specimens for genetic or molecular analysis in this trial/future use $\{33\}$}

$\mathrm{n} / \mathrm{a}$

\section{Statistical methods}

\section{Statistical methods for primary and secondary outcomes $\{20 \mathrm{a}\}$}

Statistical analysis will be performed using SPSS 20.0 and Microsoft Excel 2019 software. Each of the measures will be expressed as mean \pm standard deviation. Analysis will be performed to the measures obtained from baseline, 6-week, and 12-week of the TC training and the data between the TC \& PA groups will be compared. Two-way analysis of variance (group ' time) with repeated measures will be used for examining the effect of TC training on the primary and secondary outcomes. The participants will be analyzed based on their initially allocated groups via randomization, regardless of whether they adhere or if they received the allocated intervention. Statistical significance will be set at $P$ less than 0.05 .

\section{Interim analyses $\{21 \mathrm{~b}\}$}

$\mathrm{n} / \mathrm{a}$

\section{Methods for additional analyses (e.g., subgroup analyses) \{20b\}}

There are no planned subgroup analyses for this study. Gender difference are anticipated to be present, but these differences are not one of the primary outcomes of interest or one of the effects of TC training. We do not anticipate any gender effects to significantly contribute to the effects of TC training. Efforts have been taken to ensure that only the effects of TC training are examined, by making the intervention and control groups comparable, setting a strict age-range for the participants' age, and also using the 
individual's baseline scores for comparisons with their post-training evaluations. Therefore, we will strictly examine the potential mechanisms and training effects of TC for symptoms related specifically to PD.

\section{Methods in analysis to handle protocol non-adherence and any statistical methods to handle missing data $\{20 \mathrm{c}\}$}

All data will be thoroughly checked for completeness and that no trials are missing. Should data be missing from any of the trials, every effort will be made to locate it first, before those cases with the missing data will be omitted and analyzed for the remaining data (complete-case analysis).

\section{Plans to give access to the full protocol, participant level-data and statistical code $\{31 \mathrm{c}\}$}

Public access will be granted to the full protocol, participant-level dataset, and statistical code upon request.

\section{Oversight and monitoring}

\section{Composition of the coordinating center and trial steering committee $\{5 \mathrm{~d}\}$}

The thesis (or steering) committee of the PhD candidate, whom will be responsible for conducting and overseeing this study, will guide and provide feedback to develop and refine the details of the study. The thesis committee consists of Drs. Denis Prud'homme and Youlin Hong. This is will ensure that the quality of the candidate's work meets all academic regulations and requirements for completion of the candidate's degree. The committee will review the candidate's work for publications, travel applications, and application funds to conferences and seminars.

\section{Composition of the data monitoring committee, its role and reporting structure $\{21 \mathrm{a}\}$}

A data monitoring committee (DMC) is not needed because this role will be fulfilled by the thesis (steering) committee.

\section{Adverse event reporting and harms $\{22\}$}

Participants will be given the opportunity to take breaks or rest whenever they prefer. If the participants feel physical exerted, they will be allowed time to rest and water or other forms of rehydration methods would be provided to them. Prior to performance of the TC routine, the participants will go through a proper warm-up that will involve some basic (light) stretching, breathing, and warm-up movements for the upper and lower extremities. This would help mitigate any undesired muscle fatigue, discomfort, or soreness. To reduce the risk of falls or related injuries, the TC instructor and/or the principal investigator (PI, or a research assistant) will be available to monitor the performance abilities of each individual participant. For those deemed at a higher risk of falling, a spotter will be provided upon either request by participant or if it is evident to the instructor/PI that a spotter is needed. All serious injuries/harm resulting from either during the laboratory testing or TC intervention, PA engaged by the participants will be reported to the PI and REB of the University of Ottawa. 
Frequency and plans for auditing trial conduct $\{23\}$

Frequency and plans for auditing trial conduct will not be conducted unless required by potential funding agencies.

\section{Plans for communicating important protocol amendments to relevant parties (e.g., trial participants, ethical committees) $\{25\}$}

Due to the recent COVID-19 situation, every effort will be made to communicate important any important protocol modifications to the investigators, REB, trial participants, trial registries, journals, and steering committees.

\section{Dissemination plans $\{31$ a $\}$}

This research protocol could lead to the development of an original and innovative balance and gait training program that is tailored to individuals with PD. Other training or exercise programs can be modelled from the proposed research protocol in addition to aid, assist, or guide healthcare professionals or therapist in their development or design intervention programs for PD. By understanding the neuromuscular involvement with respect to the scientific models and principles of biomechanics in human movement analysis, we can better explain the mechanisms of PD and the potential role that TC training may provide in alleviating the symptoms that are associated with this condition. This research will help guide the development of more effective exercise programs for health promotion and fall prevention that is not restricted to the target population alone. The work involved in this study will potentially contribute to multiple publications, conference submissions, and information sessions in support groups for those with PD; this work will satisfy the requirements of a PhD degree in Rehabilitation Science or Human Kinetics.

\section{Discussion}

Different types of exercise intervention programs have been developed with limited effectiveness towards treating the movement symptoms related to PD, namely gait disorders and postural instability. Research has found that resistance training can help to improve strength after a 10 to 12-week period [63,64], but only in certain groups of muscle and are ineffective towards training overall postural control and balance. Due to the heterogeneity of the intervention and study outcomes used in these resistance training studies [74], the associated risks of injury, increased level of intensity, and longer duration of resistance training programs required to improve posture and gait are problems for those with PD. Treadmill training for 12 weeks showed an improvement in walking distance [75]. However, the operational costs needed for a body-harness or specialized equipment, assistance from a trainer or physical therapist make this form of training not feasible as a long-term option to manage PD. Balance-specific training programs are unable to improve all aspects of balance or to reduce the risk of falls, mainly because these programs rely on different exercises and activities to retrain balance and posture, with varying levels of effectiveness $[76,77]$. Strength, balance, and coordination are some aspects of postural stability that need to be 
improved, however such programs for PD would need to be highly complex and include several multimodal exercises.

TC as a "mind-body" exercise has been applied to manage the PD-related symptoms and has increased in popularity due to its rehabilitative potential. TC training shares many features as rhythmic exercises such as tango and ballroom dance that focus on: timing of the participant's foot placement; whole body coordination; awareness and attention to theirs and their partners' movement [78]. TC is a form of martial art [70-72] that has been recommended by the American Geriatric Society and British Geriatric Society for fall prevention in the elderly [73], however these are only general recommendations that are provided without the proper training protocols and guidelines for those with PD who wish to use TC as a form of therapy or exercise to maintain their physical health and wellbeing. Previous TC programs for PD have demonstrated its beneficial effects on posture, gait and mobility, but the information about the selection basis of TC movement in these studies was lacking. Therefore the mechanisms in the beneficial effects of TC training on gait and postural stability for the people with PD is unknown [74-76]. Recent evidence from studies using MRI have provided support to the beneficial effects of TC practice on brain structure and cognition function [25,77]. More research on TC intervention on gait, postural stability, and cognition function would provide a better understand the effects of practicing TC in PD and to access its role and safety as an effective form of exercise to improve quality of the life of this population.

The proposed study protocol is unique and novel. This is because firstly it is specifically and purposefully designed for PD people for improving their postural stability and gait performance. Furthermore, the biomechanics characteristics of seven TC movement have been understood that forms a foundation for the understanding of the mechanism of TC training; and lastly, but not least, with the future development of multimedia materials, tablet and mobile phone APP's, the TC intervention program would become more feasible and practical. This protocol could lead to the development of an original and innovative balance and gait training program that could help individuals with PD. We will use a full 3-D biomechanical analysis to understand the impact of TC training on movement behavior and postural strategies, and how this relates to walking performance and obstacle crossing. This study can serve as model for healthcare professionals or therapists when designing or developing future intervention programs for PD. By understanding the neuromuscular involvement of exercise through biomechanical and human movement analysis, we can better explain the mechanisms of PD and the potential role that TC training may provide in alleviating the symptoms that are associated with this condition.

Possible limitation of the study is that although TC has been shown to be gentle on the body, participants with PD may have difficulties following all demonstrations and instructions from the TC instructor. It is expected that the participants will be able to achieve the necessary level of intensity and duration that would normally be experienced during a light to moderate-intensity exercise. However, their underlying physical and health conditions will need to be carefully monitored by the TC instructor at each TC session. The participants' progress will be tracked by the researchers; however, they will not be challenged beyond their physical and mental limits during practice. 
In conclusion, the proposed study will fill the gap in the understanding the effects of TC training among those with $\mathrm{PD}$, using a biomechanical analysis to explain the potential changes in postural stability and gait. Understanding the effects of TC training, particularly in gait, postural stability, and cognition would provide an exercise option for the management of PD and improve the quality of life of this population.

\section{Trial status}

This study is in the early stages of planning as of Aug 2019. Application of the human ethics approval has been submitted and approved as of Oct 2019. Participant recruitment began in Oct 2019. The study will start May 2021 and will be completed by Dec 2021.

\section{Abbreviations}

A-P: Anterior-posterior; COM: Center of Mass; COM-COP: center of mass and center pressure; COP: Center of Pressure; H\&Y: Hoehn and Yahr; MCl: Mild Cognitive Impairment; M-L: Medial-lateral; MoCA: Montreal Cognitive Assessment; PA: physical activity; PD: Parkinson's disease; TC: Tai Chi; ROM: Range of Motion; SLO: Single-leg stance test with eyes open; SLC: Single-leg stance test with eyes open closed; TUG: Timed Up and Go Test; TMT-B: Trail Making Test Part B; UPDRS-III: Unified Parkinson's Disease Rating Scalemotor examination; WCST: Wisconsin Card Sorting Test; 3-D: three-dimensional.

\section{Declarations}

\section{Acknowledgements}

The authors would like to acknowledge the support from Parkinson's Canada for their volunteers and help with the recruitment, Ms. Sara Naghdlou for her help with the data processing, and Ms. Yiyang Chen for her help with the formatting of the article.

\section{Authors' contributions $\{31 \mathrm{~b}\}$}

$\mathrm{J}-\mathrm{XL}$ and $\mathrm{JN}$ contributed to the conception of the trial. J-XL, QZ, JN, and NL contributed to the design of the trial. J-XL and NL contributed to the drafting the manuscript. J-XL, JN, and NL contributed to the refining and submission of the manuscript. All authors have read and approved the final manuscript.

\section{Funding $\{4\}$}

There is no funding to acknowledge.

\section{Availability of data and materials $\{29\}$}

The PI will have access to the final trial dataset, there are no agreements that may limit such access for the investigators. . 
Ethics approval and consent to participate $\{24\}$

This study was approved by the Health Sciences research ethics board (REB) of the University of Ottawa (Ethics File Number: H-01-19-2284).

Consent for publication $\{32\}$

Consent Form

Title of the study: The Effects of Tai Chi Intervention on Postural Stability and Gait in People with Parkinson's Disease

Principal Investigator. Jing Xian Li — PhD
Associate Professor
School of Human Kinetics, Faculty of Health Science
University of Ottawa
200 Lees Avenue, Building E (room 020)
(613) 562-5800 ext. 2547

Co-Investigator: Nok-Yeung Law _ M.Sc.

PhD Candidate

Human Movement Biomechanics Laboratory

University of Ottawa

200 Lees Avenue, Building E (room 020)

(613) 562-5800 ext. 7358

nlaw098@uottawa.ca

\section{INVITATION TO PARTICIPATE:}

I am invited to participate in the above-mentioned research testing conducted by Dr. Jing Xian Li of the School of Human Kinetics, Faculty of Health Sciences, University of Ottawa. The research is entitled "The Effects of Tai Chi Intervention on Postural Stability and Gait in People with Parkinson's Disease (PD)." The purpose of the research is to study the effects of tai chi intervention on gait and postural stability in those with PD. 


\section{PURPOSE OF THE STUDY:}

I understand that the purpose of this study is to explore the changes in gait and postural stability among those with Parkinson's through 3 months of exercise engagement, such as tai chi and regular physical activity. From this research, we hope to understand the impact of exercise, such as tai chi practice, on gait and postural stability of those with PD. It is expected that more effective approaches for management of PD can be developed for those with PD and reduce the disease burden to them.

\section{ELIGIBILITY:}

To be able to participate in this study, I must be between ages of 50 to 75 years. I must be diagnosed with Parkinson's disease, severity ranging from stage 1 to 3 on the Hoehn \& Yahr scale. I must not have any motor fluctuations as measured by the Unified Parkinson's Disease Scale, motor sub-score (UPDRS-III). I must have stable medication use and be able to stand and walk independently. I must be available to attend or engage in physical activity 3 times/week for a 12-week period. I must not be enrolled in any other behavioral, pharmacological study or instructor led exercise programs during the duration of the study. I must not demonstrate mild cognitive impairment (mini-mental state examination < 24), have poorly cardiovascular disease symptoms during moderate exercise, poorly controlled hypertension, and any debilitating conditions that would prevent myself from engaging in physical activity for at least 60 minutes.

\section{PROTOCOL:}

I will randomly be assigned to either groups: tai chi or physical activity.

If I am enrolled in the tai chi group, I will be taught tai chi in a class size of 5 to 12 students offered in the laboratory or community center, by a certified tai chi instructor. Each tai chi class will be 60 minutes, offered 3 times per week. I am encouraged to attend at least two sessions per week for up to 12-weeks. To facilitate learning of the movements, I will be given a hard copy of the illustrated 6-form tai chi program or a 5 to 10 -minute demonstration video with the instructor.

If I am enrolled in the physical activity group, I will be required to engage in at least 60-minutes (3 times per week for 12 weeks) of regular physical activity on their own that will be documented in an activity log or journal. Some types of activities I will be allowed to engage in will include walking, jogging, stair climbing, or light stretching.

\section{PARTICIPATION:}

I will attend three sessions of test at the Human Movement Biomechanics laboratory, 200 Lees Avenue, University of Ottawa. The test will be at the beginning, 6, and 12 weeks after commencing either exercise programs, such as tai chi or regular physical activity. I will have to complete and return a form of my exercise or physical activity practicing activity every month for monitoring purposes. For my neuropsychological assessment, I will allow the researcher to assess my cognitive functioning using 
three commonly used neuropsychological assessment tests. During my movement assessment, I will wear shorts and a shirt that will be provided for motion capture and have small reflective markers placed on chosen anatomical landmarks on my body. I will have pairs of electrodes attached on my limbs for electromyography analysis. My body weight and height will be measured. My movement while walking and during obstacle crossing will be recorded using a 3-dimensional video recording system. I will perform 5 trials for each of type of movements, respectively. The data collection session will last about 2 hours.

\section{RISKS:}

There are no risks involved. The researcher has assured me that I will have enough time to practice crossing over the obstacle and warmup before formal data collection and I may request to stop at any time if I need to rest. In any case I am not able to keep my balance under these conditions I can withdraw from the study. The researcher has assured me that if there is any lingering discomfort, I can withdraw from the study at any time.

\section{DISADVANTAGES OF PARTICIPATING:}

I understand the primary disadvantage of participation will be the time required to complete and to perform data collection.

\section{BENEFITS:}

I will not benefit directly from my participation. However, I will gain a better understanding on my exercise engagement and physical activity performance. I will be able to learn how my participation in an exercise program and/or regular physical activity affect my physical capacity and wellbeing. My contribution in this research will be beneficial for the understanding of the effects of exercise in those with Parkinson's disease.

\section{CONFIDENTIALITY AND CONSERVATION OF THE DATA:}

I understand that the data collected from me will be used only to serve the research's purpose and data might be used in student theses and my confidentiality will be ensured. I will be assigned an anonymous identification code that will be used throughout the research. The data (completed consent forms, testing notes, and measurement recordings in paper form) will be stored in a locked filing cabinet in the office of the researcher at the University of Ottawa. The electronic data will be kept in the computer of the researcher and will be password protected. Only the researchers will have access to the data. I understand that any published results will be presented with complete anonymity.

The data will be conserved for a period of 5 years after the time of publishing the research results, at which time the electronic data will be deleted, and the printed data will be shredded.

\section{ANONYMITY:}


Anonymity will be assured in the following manner: a code number system will be employed during the data collection, analysis, and reporting. I will not be identified in any reports or publications.

\section{COMPENSATION:}

I will receive $\$ 15.00$ at the beginning of the session of evaluation of my postural stability and movement capacity to help cover my travel and parking costs. After the study has begun if I choose to withdraw, I can still receive the compensation. A simple meal will also be provided with to a maximum of $\$ 15$ if the biomechanics assessment goes over regular mealtime (lunch or dinner).

\section{VOLUNTARY PARTICIPATION:}

Participants who choose to withdrawal from the study will also be given the opportunity to withdraw their data.

I understand that I may ask questions regarding this study at any time and they will be answered. These questions may be addressed to Dr. Jing Xian Li (613.562.5800 ext. 2547)

\section{MORE INFORMATION ABOUT THIS STUDY:}

If I have any questions with regards to the ethical conduct of this study, I may contact the Protocol Officer for Ethics in Research, University of Ottawa at Room 154, Tabaret Hall 550 Cumberland Street, Ottawa, ON, K1N6N5, or (613) 562-5387 or ethics@uottawa.ca.

\section{CONSENT:}

I declare that I understand this project, the nature and degree of my participation and possible disadvantages and risks listed in this consent form. I have had the opportunity to ask all my questions concerning the different aspects of the study and have received responses to my satisfaction.

\section{I voluntarily agree to participate in this study.}

I agree that Dr. Jing Xian Li can keep my name and telephone number for contact me for any future study that she may be carrying out.

Yes

No

There are two copies of this consent from one of which is for me to keep.

Name of Participant 
Signature of Researcher

Date

\section{Competing interests $\{28\}$}

The authors declare that they have no competing interests.

\section{References}

1. Public Health Agency of Canada. Mapping Connections: An understanding of neurological conditions in Canada [Internet]. 2014 [cited 2016 Aug 26]. p. 1-98. Available from: http://www.phacaspc.gc.ca/publicat/cd-mc/mc-ec/index-eng.php

2. UCB Canada. Parkinson's Disease [Internet]. 2018 [cited 2018 Dec 3]. Available from: https://www.ucb-canada.ca/en/Patients/Conditions/Parkinson-s-Disease

3. Health Canada, Parkinson Society Canada. Parkinson's disease: Social and Economic Impact. 2003;(June):1-6.

4. Mille ML, Creath RA, Prettyman MG, Johnson Hilliard M, Martinez KM, MacKinnon CD, et al. Posture and locomotion coupling: A target for rehabilitation interventions in persons with Parkinson's disease. Parkinsons Dis. 2012;2012.

5. Mak MKY, Pang MYC, Mok V. Gait difficulty, postural instability, and muscle weakness are associated with fear of falling in people with Parkinson's disease. Parkinsons Dis. 2012;2012.

6. Tan D, Danoudis M, McGinley J, Morris ME. Relationships between motor aspects of gait impairments and activity limitations in people with Parkinson's disease: a systematic review. Parkinsonism Relat Disord [Internet]. 2012 Feb;18(2):117-24. Available from: http://dx.doi.org/10.1016/j.parkreldis.2011.07.014

7. Benka Wallen M, Franzen E, Nero H, Hagstromer M. Levels and Patterns of Physical Activity and Sedentary Behavior in Elderly People With Mild to Moderate Parkinson Disease. Phys Ther [Internet]. 2015 Aug 1;95(8):1135-41. Available from: http://ptjournal.apta.org/cgi/doi/10.2522/ptj.20140374

8. Hariz G-M, Forsgren L. Activities of daily living and quality of life in persons with newly diagnosed Parkinson's disease according to subtype of disease, and in comparison to healthy controls. Acta Neurol Scand. 2011;123(1):20-7.

9. Marsden CD. Parkinson's disease. JNeurolNeurosurgPsychiatry [Internet]. 1994;57:672-81. Available from: http://www.ncbi.nlm.nih.gov/pmc/articles/PMC1072968/pdf/jnnpsyc00036-0006.pdf 
10. Winogrodzka A, Wagenaar RC, Booij J, Wolters EC. Rigidity and bradykinesia reduce interlimb coordination in Parkinsonian gait. Arch Phys Med Rehabil [Internet]. 2005;86(2):183-9. Available from: http://linkinghub.elsevier.com/retrieve/pii/S0003999304012456

11. Rochester $L$, Galna B, Lord S, Burn D. The nature of dual-task interference during gait in incident Parkinson's disease. Neuroscience [Internet]. 2014;265:83-94. Available from:

http://dx.doi.org/10.1016/j.neuroscience.2014.01.041

12. Rochester L, Hetherington V, Jones D, Nieuwboer A, Willems A-MM, Kwakkel G, et al. Attending to the task: Interference effects of functional tasks on walking in Parkinson's disease and the roles of cognition, depression, fatigue, and balance. Arch Phys Med Rehabil [Internet]. 2004 Oct;85(10):1578-85. Available from: http://www.ncbi.nlm.nih.gov/pubmed/15468014

13. Menant JC, Schoene D, Sarofim M, Lord SR. Single and dual task tests of gait speed are equivalent in the prediction of falls in older people: A systematic review and meta-analysis. Ageing Res Rev [Internet]. 2014;16(1):83-104. Available from: http://dx.doi.org/10.1016/j.arr.2014.06.001

14. MacAulay RK, Allaire TD, Brouillette RM, Foil HC, Bruce-Keller AJ, Han H, et al. Longitudinal assessment of neuropsychological and temporal/spatial gait characteristics of elderly fallers: Taking it all in stride. Front Aging Neurosci. 2015;7(FEB):1-7.

15. Allali G, Launay CP, Blumen HM, Callisaya ML, De Cock AM, Kressig RW, et al. Falls, Cognitive Impairment, and Gait Performance: Results From the GOOD Initiative. J Am Med Dir Assoc [Internet]. 2017;18(4):335-40. Available from: http://dx.doi.org/10.1016/j.jamda.2016.10.008

16. Hausdorff JM, Rios DA, Edelberg HK. Gait variability and fall risk in community-living older adults: A 1-year prospective study. Arch Phys Med Rehabil. 2001;82(8):1050-6.

17. Latt MD, Menz HB, Fung VS, Lord SR. Acceleration patterns of the head and pelvis during gait in older people with Parkinson's disease: A comparison of fallers and nonfallers. Journals Gerontol - Ser A Biol Sci Med Sci. 2009;64(6):700-6.

18. McNeely ME, Duncan RP, Earhart GM. Medication improves balance and complex gait performance in Parkinson disease. Gait Posture [Internet]. 2012;36(1):144-8. Available from: http://dx.doi.org/10.1016/j.gaitpost.2012.02.009

19. Wood BH, Bilclough JA, Bowron A, Walker RW. Incidence and prediction of falls in Parkinson's disease: a prospective multidisciplinary study. J Neurol Neurosurg Psychiatry [Internet]. 2002 Jun;72(6):721-5. Available from: http://www.ncbi.nlm.nih.gov/pubmed/12023412

20. Zhou J, Yin T, Gao Q, Yang XC. A Meta-Analysis on the Efficacy of Tai Chi in Patients with Parkinson's Disease between 2008 and 2014. Evidence-Based Complement Altern Med [Internet]. 2015;2015((Zhou J., zhouji_22@hotmail.com) Department of Tuina, Guang'Anmen Hospital, China 
Academy of Chinese Medical Sciences, Beijing, China):1-9. Available from: http://www.epistemonikos.org/documents/eda156c8cdaa31cfad9af9bab78f920c4e8c94e1

21. Yang Y, Qiu WQ, Hao YL, Lv ZY, Jiao SJ, Teng JF. The Efficacy of Traditional Chinese Medical Exercise for Parkinson's Disease: A Systematic Review and Meta-analysis. PLoS One [Internet]. 2015;10(4):e0122469. Available from: http://dx.plos.org/10.1371/journal.pone.0122469

22. Petzinger GM, Fisher BE, McEwen S, Beeler J a, Walsh JP, Jakowec MW. Exercise-enhanced neuroplasticity targeting motor and cognitive circuitry in Parkinson's disease. Lancet Neurol [Internet]. 2013;12(7):716-26. Available from: http://ovidsp.ovid.com/ovidweb.cgi?

$\mathrm{T}=\mathrm{JS} \& C S C=Y \& N E W S=N \& P A G E=$ fulltext $\& D=$ medl\&AN=23769598

23. van der Kolk NM, King LA. Effects of exercise on mobility in people with Parkinson's disease. Mov Disord [Internet]. 2013 Sep 15 [cited 2016 Feb 8];28(11):1587-96. Available from: http://www.ncbi.nlm.nih.gov/pubmed/24132847

24. Zhang T-YY, Hu Y, Nie Z-YY, Jin R-XX, Chen F, Guan Q, et al. Effects of Tai Chi and Multimodal Exercise Training on Movement and Balance Function in Mild to Moderate Idiopathic Parkinson Disease. Am J Phys Med Rehabil [Internet]. 2015 Oct;94(10 Suppl 1):921-9. Available from: http://content.wkhealth.com/linkback/openurl?sid=WKPTLP:landingpage\&an=00002060-20151000100011

25. Yao J, Song Q, Zhang K, Hong Y, Li W, Mao D, et al. The effect of Tai Chi practice on brain white matter structure: a diffusion tensor magnetic resonance imaging study. Res Sports Med [Internet]. 2018 Jul 27;27(1):1-10. Available from: https://doi.org/10.1080/15438627.2018.1502184

26. Chang S, Zhou J, Hong Y, Sun W, Cong Y, Qin M, et al. Effects of 24-week Tai Chi exercise on the knee and ankle proprioception of older women. Res Sports Med [Internet]. 2016 Jan 2 [cited 2017 Feb 21];24(1):84-93. Available from: http://www.tandfonline.com/doi/full/10.1080/15438627.2015.1126281

27. Law N-Y, Li JX. The Temporospatial and Kinematic Characteristics of Typical Tai Chi Movements: Repulse Monkey and Wave-hand in Cloud. Res Sport Med [Internet]. 2014 Apr 3 [cited 2016 Mar 30];22(2):111-23. Available from: http://www.ncbi.nlm.nih.gov/pubmed/24650332

28. Tsang WW, Wong VS, Fu SN, Hui-Chan CW. Tai Chi Improves Standing Balance Control under Reduced or Conflicting Sensory Conditions. Arch Phys Med Rehabil. 2004;85(1):129-37.

29. Li JX, Law N-Y. Kinetics of the lower limb during two typical Tai Chi movements in the elderly. Res Sports Med [Internet]. 2018 Jan 2;26(1):112-23. Available from: https://www.tandfonline.com/doi/full/10.1080/15438627.2017.1393753

30. Li JX, Xu DQ, Hong Y. Changes in muscle strength, endurance, and reaction of the lower extremities with Tai Chi intervention. J Biomech [Internet]. 2009 May [cited 2016 Mar 30];42(8):967-71. Available 
from: http://www.sciencedirect.com/science/article/pii/S0021929009001213

31. Xu DQ, Li JX, Hong Y. Effects of long term Tai Chi practice and jogging exercise on muscle strength and endurance in older people. Br J Sports Med [Internet]. 2006 Jan [cited 2016 Mar 30];40(1):50-4; discussion 50-4. Available from: http://bjsm.bmj.com/content/40/1/50.short

32. Wang S-JJ, Xu D-QQ, Li J-XX. Effects of regular Tai Chi practice and jogging on neuromuscular reaction during lateral postural control in older people. Res Sport Med [Internet]. 2017 Jan 2;25(1):111-7. Available from: http://dx.doi.org/10.1080/15438627.2016.1258649

33. Fong S-MM, Ng GY. The Effects on Sensorimotor Performance and Balance With Tai Chi Training. Arch Phys Med Rehabil [Internet]. 2006;87(1):82-7. Available from: http://ovidsp.ovid.com/ovidweb.cgi? $\mathrm{T}=\mathrm{JS} \& \mathrm{PAGE}=$ reference\&D$=$ med $5 \& \mathrm{NEWS}=\mathrm{N} \& \mathrm{AN}=16401443$

34. Tsang WWNN, Hui-Chan CWYY. Effects of Tai Chi on Joint Proprioception and Stability Limits in Elderly Subjects. Med Sci Sport Exerc [Internet]. 2003;35(12):1962-71. Available from:

http://content.wkhealth.com/linkback/openurl?sid=WKPTLP:landingpage\&an=00005768-20031200000003

35. Mitchell SL, Collin JJ, De Luca CJ, Burrows A, Lipsitz LA. Open-loop and closed-loop postural control mechanisms in Parkinson's disease: increased mediolateral activity during quiet standing. Neurosci Lett. 1995;197(2):133-6.

36. Frenklach A, Louie S, Koop MM, Bronte-Stewart HM. Excessive postural sway and the risk of falls at different stages of parkinson's disease. Mov Disord. 2009;24(3):377-85.

37. Matinolli M, Korpelainen JT, Korpelainen R, Sotaniemi KA, Virranniemi M, Myllyl?? V V. Postural sway and falls in Parkinson's disease: A regression approach. Mov Disord. 2007;22(13):1927-35.

38. Fuzhong L, Harmer P, Fitzgerald K, Eckstrom E, Stock R, Galver J, et al. Tai Chi and Postural Stability in Patients with Parkinson's Disease. N Engl J Med. 2012;366:511-9.

39. Yang Y, Hao Y lei, Tian W jing, Gong L, Zhang K, Shi Q guang, et al. The effectiveness of Tai Chi for patients with Parkinson's disease: study protocol for a randomized controlled trial. Trials [Internet]. 2015 Mar;16(1):111. Available from: http://www.trialsjournal.com/content/16/1/111

40. Xu D, Hong Y, Li J, Chan K. Effect of tai chi exercise on proprioception of ankle and knee joints in old people. Br J Sports Med [Internet]. 2004 Feb [cited 2016 Mar 30];38(1):50-4. Available from: https://bjsm.bmj.com/content/38/1/50.full

41. Hoehn, M.M., Yahr MD. Parkinsonism: onset, progression, and mortality. Neurology. 1967;

42. Fahn S, Elton R. Members of the UPDRS development committee. Recent Dev Park Dis. 1987;2(J):153. 
43. Hoops S, Nazem S, Siderowf AD, Duda JE, Xie SX, Stern MB, et al. Validity of the MoCA and MMSE in the detection of $\mathrm{MCl}$ and dementia in Parkinson disease. Neurology. 2009;73(21):1738-45.

44. Mark B-S. Combined Tai-Chi Chuan. Boston: Chinese Wushu Research Institute; 1979.

45. Mao DW, Hong Y, Li JX. Characteristics of foot movement in Tai Chi exercise. Phys Ther [Internet]. 2006 Feb 1;86(2):215-22. Available from:

https://academic.oup.com/ptj/article/86/2/215/2805076/Characteristics-of-Foot-Movement-in-Tai-Chi

46. Law N-Y, Li J. The Temporospatial, Kinematics, and Electromyography features of seven typical Tai Chi forms [in press]. Gait Posture. 2019;

47. Law N-Y, Li JX. Tai Chi Intervention Program for Improving Postural Stability and Gait in People with Parkinson's Disease: a study protocol. In: Conference proceedings: . Annual International Conference of the World Conference on Movement and Cognition Annual Conference. 2019.

48. Hass CJ, Waddell DE, Fleming RP, Juncos JL, Gregor RJ. Gait Initiation and Dynamic Balance Control in Parkinson's Disease. Arch Phys Med Rehabil [Internet]. 2005 Nov;86(11):2172-6. Available from: https://linkinghub.elsevier.com/retrieve/pii/S0003999305005198

49. Hamacher D, Singh NB, Van Dieën JH, Heller MO, Taylor WR. Kinematic measures for assessing gait stability in elderly individuals: A systematic review. J R Soc Interface. 2011;8(65):1682-98.

50. Chang Y-TT, Huang C-FF, Chang J-HH. The Effect of Tai Chi Chuan on Obstacle Crossing Strategy in Older Adults. Res Sport Med [Internet]. 2015 Jul 3 [cited 2017 Feb 21];23(3):315-29. Available from: https://apps.webofknowledge.com/full_record.do?

product=WOS\&search_mode $=$ GeneralSearch\&qid $=27 \& S I D=T 2 D g D m 048 f b L m a O S z W y \& p a g e=1 \& d o c=3$

51. Li X. Dynamic Postural Stability of Old Tai Chi Practitioners during Obstacle-crossing [Internet]. University of Ottawa; 2016 [cited 2018 Nov 5]. Available from:

https://www.ruor.uottawa.ca/handle/10393/35112

52. Chou LLS, Kaufman KRKKR, Hahn MEMME, Brey RHR. Medio-lateral motion of the center of mass during obstacle crossing distinguishes elderly individuals with imbalance. Gait Posture [Internet]. 2003 [cited 2017 May 6];18(3):125-33. Available from:

http://www.sciencedirect.com/science/article/pii/S096663620200067X

53. Fahn S, Elton R. UNIFIED PARKINSON'S DISEASE RATING SCALE. In: Fahn S, Marsden CD, Calne D, Goldsten M, editors. Recent Development in Parkinson's Disease. Vol 2. Florham Park, NJ; 1987. p. 15 3163, 293-304.

54. Chomiak T, Pereira FV, Hu B. The Single-Leg-Stance Test in Parkinson's Disease. J Clin Med Res [Internet]. 2015;7(3):182-5. Available from: http://www.jocmr.org/index.php/JOCMR/article/view/1878 
55. Springer BA, Marin R, Cyhan T, Roberts H, Gill NW. Normative values for the unipedal stance test with eyes open and closed. J Geriatr Phys Ther. 2007;30(1):8-15.

56. Lysack CL. Household and Neighborhood Safety, Mobility. In: Handbook of Assessment in Clinical Gerontology [Internet]. Elsevier; 2010. p. 619-46. Available from:

https://linkinghub.elsevier.com/retrieve/pii/B9780123749611100235

57. Morris S, Morris ME, lansek R. Reliability of measurements obtained with the Timed "Up \& Go" test in people with Parkinson disease. Phys Ther [Internet]. 2001 Feb;81(2):810-8. Available from: http://www.ncbi.nlm.nih.gov/pubmed/11175678

58. Muslimović D, Post B, Speelman JD, De Haan RJ, Schmand B. Cognitive decline in Parkinson's disease: a prospective longitudinal study. Vol. 15, Journal of the International Neuropsychological Society. 2009. 426-437 p.

59. Duchesne C, Lungu O, Nadeau A, Robillard MEE, Boré A, Bobeuf F, et al. Enhancing both motor and cognitive functioning in Parkinson's disease: Aerobic exercise as a rehabilitative intervention. Brain Cogn [Internet]. 2015 Oct;99:68-77. Available from: http://dx.doi.org/10.1016/j.bandc.2015.07.005

60. Olivera-Souza RD, Moll J, Passman LJ, Cunha FC, Paes F, Adriano M V, et al. Trail making and cognitive set-shifting. Arq Neuropsiquiatr [Internet]. 2000 Sep;58(3B):826-9. Available from: http://www.scielo.br/scielo.php?script=sci_arttext\&pid=S0004-282X2000000500006\&lng=en\&tlng=en

61. Perrochon A, Kemoun G. The Walking Trail-Making Test is an early detection tool for mild cognitive impairment. Clin Interv Aging [Internet]. 2014 Jan;9:111. Available from: papers://58312f1a-429f-4e798aa2-9c8b010ae96e/Paper/p927

62. Watson G, Leverenz JB. Profile of Cognitive Impairment in Parkinson's Disease. Brain Pathol [Internet]. 2010;20(3):640-645. Available from: http://onlinelibrary.wiley.com/doi/10.1111/j.17503639.2010.00373.x/full

63. Chan RCK, Shum D, Toulopoulou T, Chen EYH. Assessment of executive functions: Review of instruments and identification of critical issues. Arch Clin Neuropsychol. 2008;23(2):201-16.

64. Galtier I, Nieto A, Lorenzo JN, Barroso J. Cognitive Impairment in Parkinson's Disease: More than a Frontostriatal Dysfunction. Span J Psychol [Internet]. 2014 Oct 28;17(2014):E68. Available from: https://www.cambridge.org/core/product/identifier/S1138741614000699/type/journal_article

65. Faria $\mathrm{C}$ de $\mathrm{A}$, Veiga $\mathrm{H}$, Alves D, Charchat-Fichman H, Alves HVD, Charchat-Fichman $\mathrm{H}$. The most frequently used tests for assessing executive functions in aging. Dement Neuropsychol [Internet]. 2015;9(2):149-55. Available from: http://www.scielo.br/scielo.php?script=sci_arttext\&pid=S1980$57642015000200149 \&$ lng $=$ en\&nrm=iso\&tlng=en 
66. Litvan I, Goldman JG, Tröster Al, Schmand BA, Weintraub D, Petersen RC, et al. Diagnostic criteria for mild cognitive impairment in Parkinson's disease: Movement Disorder Society Task Force guidelines. Mov Disord [Internet]. 2012 Mar;27(3):349-56. Available from:

http://www.pubmedcentral.nih.gov/articlerender.fcgi?

artid $=3641655 \&$ tool $=$ pmcentrez\&rendertype $=$ abstract

67. Gao Q, Leung A, Yang Y, Wei Q, Guan M, Jia C, et al. Effects of Tai Chi on balance and fall prevention in Parkinson's disease: a randomized controlled trial. Clin Rehabil [Internet]. 2014 Aug;28(8):748-53. Available from: http://www.ncbi.nlm.nih.gov/pubmed/24519923

68. Cheon S-MM, Chae B-KK, Sung H-RR, Lee GC, Kim JW. The efficacy of exercise programs for Parkinson's disease: Tai Chi versus combined exercise. J Clin Neurol [Internet]. 2013;9(4):237-43. Available from: http://www.pubmedcentral.nih.gov/articlerender.fcgi? artid $=3840134 \&$ tool $=$ pmcentrez\&rendertype $=$ abstract

69. Li F, Harmer P, Fitzgerald K, Eckstrom E, Stock R, Galver J, et al. Tai Chi and Postural Stability in Patients with Parkinson's Disease. N Engl J Med [Internet]. 2012 Feb 9;366(6):511-9. Available from: http://www.nejm.org/doi/abs/10.1056/NEJMoa1107911

70. Kim H-DD, Jae HD, Jeong JH. Tai Chi Exercise can Improve the Obstacle Negotiating Ability of People with Parkinson's Disease: A Preliminary Study. J Phys Ther Sci [Internet]. 2014;26(7):1025-30. Available from: http://www.pubmedcentral.nih.gov/articlerender.fcgi? artid $=4135189 \&$ tool $=$ pmcentrez\&rendertype $=$ abstract

71. Paterson DH, Jones GR, Rice CL. Ageing and physical activity: evidence to develop exercise recommendations for older adults. Can J public Heal Rev Can sant?? publique. 2007;

72. Dibble LE, Hale TF, Marcus RL, Droge J, Gerber JP, LaStayo PC. High-intensity resistance training amplifies muscle hypertrophy and functional gains in persons with Parkinson's disease. Mov Disord [Internet]. 2006;21(9):1444-52. Available from: http://ovidsp.ovid.com/ovidweb.cgi? $T=J S \& C S C=Y \& N E W S=N \& P A G E=$ fulltext $\& D=\operatorname{med} 5 \& A N=16773643$

73. Hirsch MA, Toole T, Maitland CG, Rider RA. The effects of balance training and high-intensity resistance training on persons with idiopathic Parkinson's disease. Arch Phys Med Rehabil. 2003;84(8):1109-17.

74. Cruickshank TM, Reyes AR, Ziman MR. A Systematic Review and Meta-Analysis of Strength Training in Individuals With Multiple Sclerosis Or Parkinson Disease. Medicine (Baltimore). 2015;94(4).

75. Shulman LM, Katzel LI, Ivey FM, Sorkin JD, Favors K, Anderson KE, et al. Randomized Clinical Trial of 3 Types of Physical Exercise for Patients With Parkinson Disease. JAMA Neurol [Internet]. 2013 Feb 1;70(2):183. Available from: http://ovidsp.ovid.com/ovidweb.cgi? $\mathrm{T}=\mathrm{JS} \& C S C=Y \& N E W S=N \& P A G E=$ fulltext $\& D=$ medc\&AN=23128427 
76. Smania N, Corato E, Tinazzi M, Stanzani C, Fiaschi A, Girardi P, et al. Effect of Balance Training on Postural Instability in Patients With Idiopathic Parkinson's Disease. Neurorehabil Neural Repair [Internet]. 2010 Jan 1 [cited 2016 Mar 14];24(9):826-34. Available from:

http://nnr.sagepub.com/content/24/9/826.short

77. Combs SA, Diehl MD, Chrzastowski C, Didrick N, McCoin B, Mox N, et al. Community-based group exercise for persons with Parkinson disease: A randomized controlled trial. NeuroRehabilitation. 2013;32(1):117-24.

78. Hackney ME, Wolf SL. Impact of Tai Chi Chu'an Practice on Balance and Mobility in Older Adults. J Geriatr Phys Ther [Internet]. 2014;37(3):127-35. Available from: http://content.wkhealth.com/linkback/openurl?sid=WKPTLP:landingpage\&an=00139143-20140700000005

79. Li F. Transforming traditional Tai Ji Quan techniques into integrative movement therapy-Tai Ji Quan: Moving for Better Balance. J Sport Heal Sci [Internet]. 2014 Mar 28;3(1):9-15. Available from: http://dx.doi.org/10.1016/j.jshs.2013.11.002

80. Solloway MR, Taylor SL, Shekelle PG, Miake-Lye IM, Beroes JM, Shanman RM, et al. An evidence map of the effect of Tai Chi on health outcomes. Syst Rev. 2016 Jul;5(1):126.

81. Wolf SL, Barnhart HX, Ellison GL, Coogler CE, Barnhart. The Effect of Tai Chi Quan and Computerized Balance Training on Postural Stability in Older Subjects. Phys Ther [Internet]. 1997;77(4):371-81. Available from: https://academic.oup.com/ptj/article/2633149/The

82. Drootin M. Summary of the Updated American Geriatrics Society/British Geriatrics Society Clinical Practice Guideline for Prevention of Falls in Older Persons. J Am Geriatr Soc [Internet]. 2011 Jan;59(1):148-57. Available from: http://doi.wiley.com/10.1111/j.1532-5415.2010.03234.x

83. Li F, Harmer P, Fitzgerald K, Eckstrom E, Stock R, Galver J, et al. Tai chi and postural stability in patients with Parkinson's disease. N Engl J Med. 2012;366(6):511-9.

84. Hackney ME, Earhart GM. Tai Chi improves balance and mobility in people with Parkinson disease. Gait Posture [Internet]. 2008 Oct;28(3):456-60. Available from:

http://linkinghub.elsevier.com/retrieve/pii/S0966636208000647

85. Tao J, Liu J, Egorova N, Chen X, Sun S, Xue X, et al. Increased Hippocampus-Medial Prefrontal Cortex Resting-State Functional Connectivity and Memory Function after Tai Chi Chuan Practice in Elder Adults. Front Aging Neurosci [Internet]. 2016 Feb 16;8. Available from:

http://journal.frontiersin.org/Article/10.3389/fnagi.2016.00025/abstract

\section{Figures}


Assess for eligibility $(n=40)$
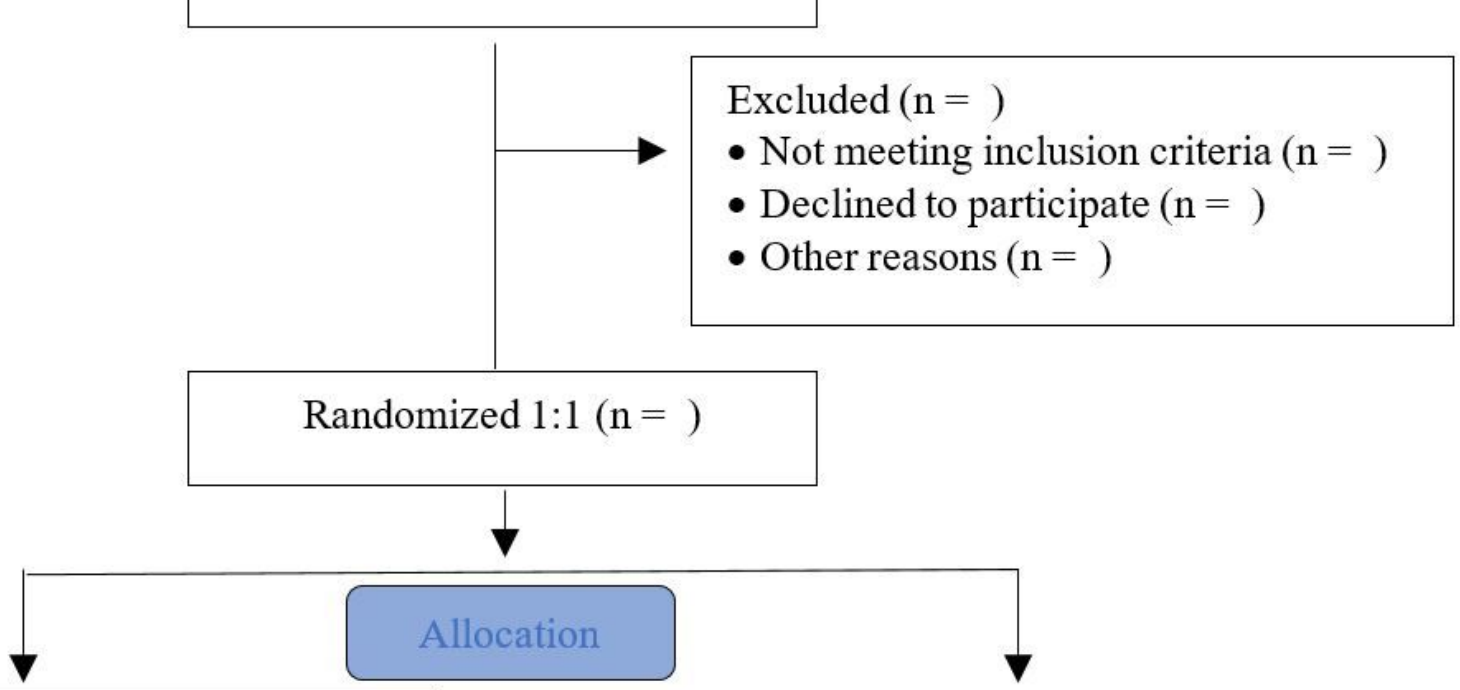

Allocated to TC intervention group $(\mathrm{n}=)$

- Received allocated intervention $(\mathrm{n}=)$

- Did not receive allocated intervention (give reasons) $(\mathrm{n}=$ )

Allocated to typical PA group $(\mathrm{n}=)$

- Received allocated intervention $(\mathrm{n}=)$

- Did not receive allocated intervention (give reasons) $(\mathrm{n}=$ )

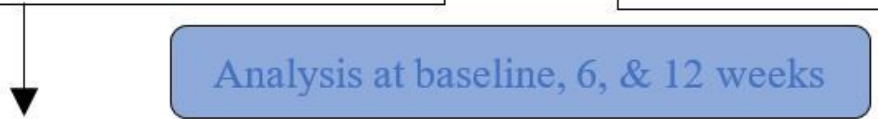

Analyzed $(\mathrm{n}=)$

- Excluded from analysis (give reasons) $(\mathrm{n}=$ )

Analyzed $(\mathrm{n}=)$

- Excluded from analysis (give reasons) $(n=)$

\section{Figure 1}

Flow diagram of TC study with design and illustration of experimental and control 


\section{Wave-Hand-like-Cloud}

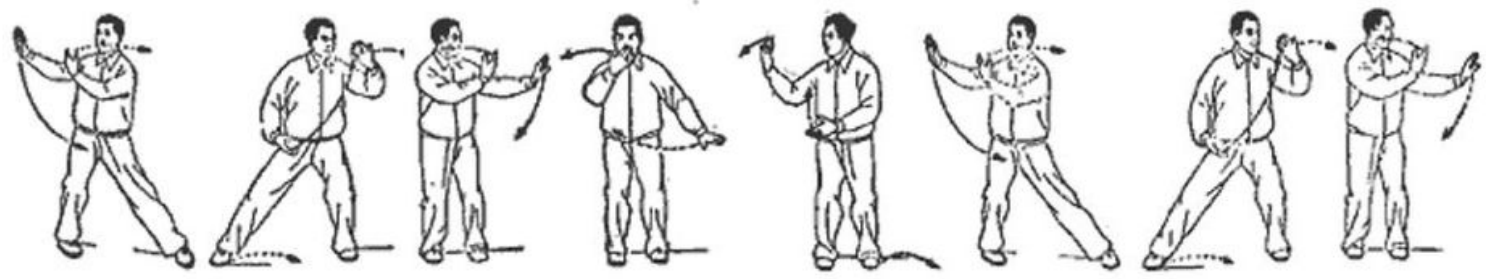

\section{Brush Knee Twist}

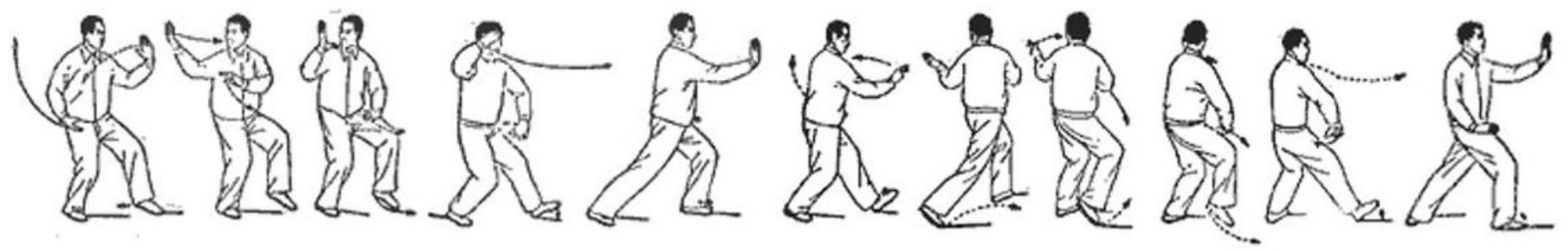

\section{Repulse Monkey}

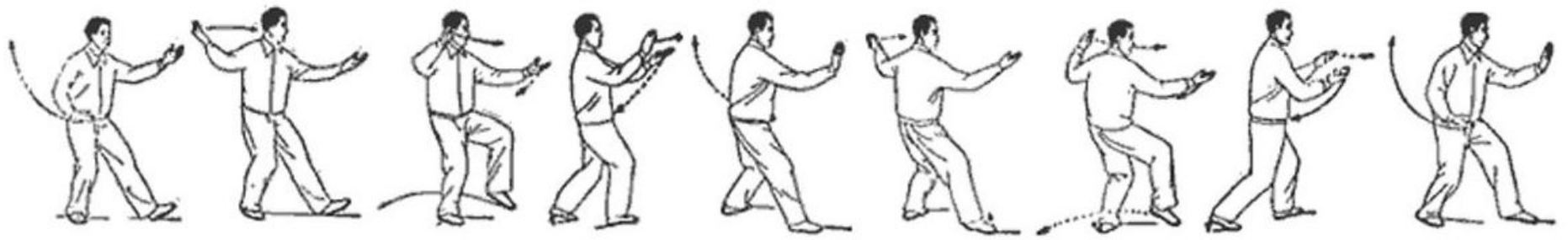

\section{Part the Wild Horse's Mane}

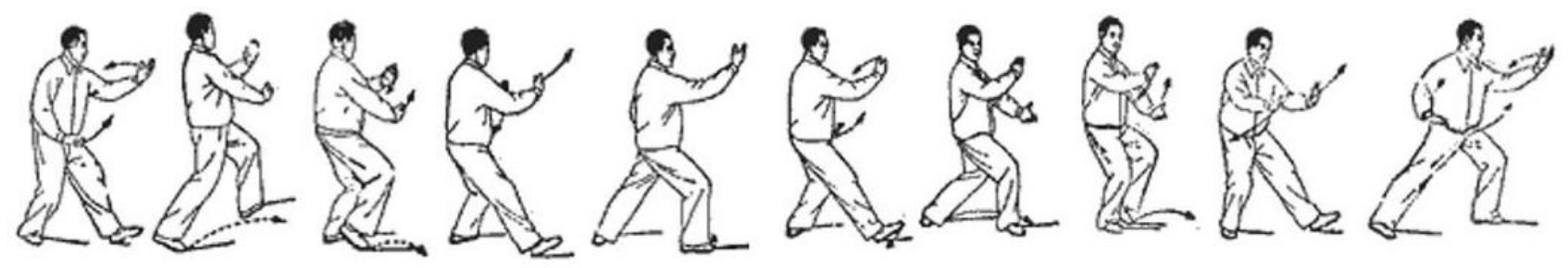

Figure 2

Illustration of four dynamic forms from the proposed simplified 7-form TC program 


\begin{tabular}{|c|c|c|c|c|c|c|}
\hline & \multicolumn{6}{|c|}{ STUDY PERIOD } \\
\hline & \multirow{2}{*}{$\begin{array}{c}\text { Enrolment } \\
-t_{1}\end{array}$} & \multirow{2}{*}{$\begin{array}{c}\text { Allocation } \\
0\end{array}$} & \multicolumn{3}{|c|}{ Post-allocation } & \multirow{2}{*}{$\begin{array}{c}\text { Close-out } \\
t_{x}\end{array}$} \\
\hline $\begin{array}{r}-\mathrm{t} 1=\text { Jan } 2021 \\
\mathrm{t} 1=\text { May } 2021 \\
\mathrm{t} 2=\text { Jun } 2021 \\
\mathrm{t} 3=\text { Jul } 2021 \\
\mathrm{tx}=\text { = Dec } 2021\end{array}$ & & & $t_{1}$ & $t_{2}$ & $t_{3}$ & \\
\hline \multicolumn{7}{|l|}{ ENROLMENT: } \\
\hline Eligibility screen & $\mathrm{X}$ & & & & & \\
\hline Informed consent & $\mathrm{X}$ & & & & & \\
\hline Allocation & & $\mathrm{X}$ & & & & \\
\hline \multicolumn{7}{|l|}{ INTERVENTIONS: } \\
\hline \multicolumn{7}{|l|}{ TC training } \\
\hline Regular PA & & & $x$ & & $x$ & \\
\hline \multicolumn{7}{|l|}{ ASSESSMENTS: } \\
\hline $\begin{array}{r}\text { Demographic } \\
\text { details and }\end{array}$ & X & X & & & & \\
\hline $\begin{array}{r}\text { Gait and Dynamic } \\
\text { Postural Stability } \\
\text { parameters }\end{array}$ & & & $\mathrm{X}$ & $\mathrm{x}$ & $\mathrm{x}$ & \\
\hline $\begin{array}{r}\text { Clinical } \\
\text { assessments }\end{array}$ & & & $X$ & $\mathrm{X}$ & $\mathrm{X}$ & \\
\hline $\begin{array}{r}\text { Cognitive } \\
\text { Assessments }\end{array}$ & & & $X$ & $\mathrm{X}$ & $\mathrm{X}$ & \\
\hline Exit Questionnaire & & & & & & $x$ \\
\hline
\end{tabular}

Figure 3

Schedule of enrolment, intervention, and assessment for proposed TC intervention study involving those with Parkinson's disease. 